\title{
PREECLAMPSIA SEVERA DE APARICIÓN TEMPRANA: CARACTERÍSTICAS CLÍNICO EPIDEMIOLÓGICAS EN LA UNIDAD DE CUIDADOS INTENSIVOS MATERNO DEL INMP AGOSTO DEL 2014 A SETIEMBRE DEL 2018
}

\author{
Walter J. De la Peña Meniz ${ }^{1,2}$, Alberto Díaz Seminario ${ }^{1,2}$, Ronald Meza Salcedo ${ }^{1,2}$, Hernán Sandoval Manrique ${ }^{1,2}$, \\ Julio Cano Loayza ${ }^{1,2}$, Alfredo Castillo Gozzer ${ }^{1,2}$, Kenny Villalobos Corrales ${ }^{1,2}$, Lorena Manrique ${ }^{1,2}$.
}

\begin{abstract}
RESUMEN
Objetivo: conocer las características epidemiológicas clínicas y patológicas de la Preeclampsia severa (PES) de aparición temprana y la PES de aparición tardía de pacientes admitidas a la Unidad de Cuidados Intensivos Maternos (UCIM) del Instituto Nacional Materno Perinatal (INMP). Materiales y métodos: se realizó un estudio descriptivo transversal comparativo en el que se revisaron las historias clínicas desde EL primero de agosto del 2014 hasta el 30 de Setiembre del 2018 obteniendo 332 registros de pacientes con PES de aparición Temprana y 325 registros de pacientes con PES de aparición tardía.Resultados: La edad promedio de las pacientes con diagnóstico de PES de aparición temprana fue 27.84 años [IC 26.88- 28.81 ( $p: 0.72$ )], el promedio de estancia hospitalaria fue mayor en las pacientes con PES de aparición temprana ( $p$ $0.006)$. Los recién nacidos mostraron diferencias significativas con respecto al peso $(p=0.000)$, la talla $(p=0.000)$; el APGAR al minuto fue significativamente menor en el grupo de aparición temprana $(p=0.000)$. No se encuentra diferencia significativa entre la PES de aparición temprana y la eclampsia $(p=0.481)$. Conclusión: No observamos diferencias significativas entre PES de aparición temprana y eclampsia. Los recién nacidos, presentan peso, talla y APGAR menores en el grupo de aparición temprana que el grupo de aparición tardía.
\end{abstract}

Palabras Clave: Preeclampsia severa; Características clínicas; Características epidemiológicas.(Fuente: DeCS BIREME).

\section{SEVERE PREVENTION OF EARLY APPEARANCE: EPIDEMIOLOGICAL CLINICAL CHARACTERISTICS IN THE MATERNAL INTENSIVE CARE UNIT OF INMP AUGUST 2014 TO SEPTEMBER 2018}

\begin{abstract}
Objective: to acknowledge the pathological, clinical and epidemiological characteristics of early-onset severe preeclampsia and late-onset severe preeclampsia in patients admitted in the maternal intensive care unit (MICU) of the maternal perinatal national institute (INMP). Materials and methods: We develop a comparative transversal descriptive study. Clinical charts were reviewed from August first of 2014 until September thirty of 2018 getting 332 charts of early-onset preeclampsia and 325 charts of the late-onset type. Results: The mean age of the patients with early-onset severe preeclampsia was 27.84 years (IC 26.88- 28.81) (p: 0.72), the mean for hospital stay was higher in the patients with the early-onset type ( $p: 0.006)$. The new born showed statistical difference in weight $(p=0.000)$, height $(p=0.000)$ and the APGAR score was lower in the early-onset group $(p=0.000)$. We did not found difference statistically significative between early-onset severe preeclampsia and eclampsia $(p=0.481)$. Conclusion: we did not found differences between early onset preeclampsia and eclampsia. New born had lower weight, height and APGAR score in the early-onset group than in the late-onset group.
\end{abstract}

Key Words: Severe preeclampsia; Clinical features; Epidemiological characteristics. (Source DeCS BIREME).

\section{INTRODUCCIÓN}

La Preeclampsia es una patología de origen vascular que compromete la evolución natural del embarazo. Afecta 5 a $8 \%$ de todos los embarazos ${ }^{1}$. Las manifestaciones se presentan después de las 20 semanas de gestación. Es más frecuente en países menos favorecidos económicamente ${ }^{2}$.
La preeclampsia tiene relación directa con la mortalidad materna cada año más de medio millón de mujeres mueren por causas relacionadas con el embarazo, solo el $1 \%$ de las muertes maternas ocurren en países desarrollados ${ }^{3}$. La preeclampsia está definida como gestante de más de 20 semanas de gestación con presencia de hipertensión mayor de 140/90 mmHg y proteinuria (>300 mg/24 Hrs). En caso de severidad puede desarrollar alteración

Instituto Nacional Materno Perinatal de Lima, Perú

Unidad de Cuidados Intensivos Materno

Citar como: De La Peña WJ, Díaz A, Meza R, Sandoval H, Cano J, Castillo A, Villalobos K, Manrique L. Preeclampsia severa de aparición temprana: Características clínico epidemiológicas en la Unidad de Cuidados Intensivos Materno del INMP agosto del 2014 a setiembre del 2018 . Rev Peru Investig Matern Perinat 2020; 9(1): 28-32.

DOI https://doi.org/10.33421/inmp.2020181

Recibido: 15-02-20 Aprobado: 12-03-20 
hepática con presencia de hemolisis y trombocitopenia (síndrome HELLP), eclampsia, insuficiencia respiratoria, insuficiencia renal ${ }^{4}$.

En la década de los 80 aparecieron artículos que describían características clínico y fetales distintos en pacientes con preeclampsia de aparición temprana la cual se definió como la preeclampsia que se desarrolla ${ }^{5}$ antes de las 34 semanas de gestación mientras la preeclampsia de aparición tardía la cual se desarrolla después de las 34 semanas de gestación ${ }^{6}$, en estos artículos se infiere que la pes de aparición temprana tiene su origen en el feto y la placenta mientras que la preeclampsia tardía tiene su origen en la madre ${ }^{6,7}$ la preeclampsia de aparición temprana es la forma más severa y está asociada con retardo del crecimiento intrauterino (RCIU), patología fetal y circulación sanguínea uterina, placenta de pequeño tamaño, parto pretermino, morbilidad y mortalidad neonatal, la frecuencia de presentación es 5-20\%. La preeclampsia de aparición tardía es aproximadamente $75-80 \%$ de todos los casos de preeclampsia, está asociada con morbilidad materna (síndrome metabólico, tolerancia a la glucosa alterada, obesidad, dislipidemia e hipertensión crónica) ${ }^{8}$. Se relaciona a la PES de aparición temprana como una entidad independiente, relacionada a patología placentaria con mayores complicaciones maternas mientras que la PES de aparición tardía sería una entidad relacionada a un origen materno con menores complicaciones y alteraciones fetales sobre todo de macrosomia ${ }^{5}$.

\section{MATERIALES Y MÉTODOS}

El presente corresponde a un estudio descriptivo transversal comparativo, se revisaron las historias clínicas de las pacientes admitidas a la Unidad de Cuidados Intensivos Materno del INMP, desde el $1^{\circ}$ de Agosto del 2014 al 31 de agosto del 2018.

Se incluyeron 657 registros de pacientes con enfermedad hipertensiva del embarazo (EHE). Las variables cuantitativas edad, peso, talla, IMC, menarquia, edad de primera

$R S$, edad gestacional, peso del recién nacido (RN), Talla del RN, APGAR al minuto, hemoglobina, TGO, TGP, plaquetas, PAS, PAD, PAM, Frecuencia Cardiaca, Creatinina, y estancia hospitalaria. Las variables cualitativas estado civil, primipaternidad, cesárea, uso de hemoderivados, la edad reagrupada.

Las variables cuantitativas se expresaron como media +/- desviación estándar las variables cualitativas como frecuencia (porcentaje), la comparación entre grupos se realizó en el caso de las variables cuantitativas con el test de la $\mathrm{T}$ de Student (previa prueba de normalidad con el Shapiro Wilks) o en su caso con el test no paramétrico $U$ de Mann Whitney, en el caso de las variables cualitativas se utilizó el test de Chi cuadrado o la prueba de exacta de Fisher.
En todos los casos se consideró la existencia de significación estadística a aquella con $p<0.05$ y el intervalo de confianza se estableció para el $95 \%$ de los casos. El análisis estadístico se realizó utilizando el paquete estadístico STATA versión 14.

\section{RESULTADOS}

La media de edad fue de $28.22+/-7.61$ años , el peso promedio fue de $62.41 \mathrm{~kg}+/-11.75$, la talla promedio 1.53 $\mathrm{mts}+/-0.06$, el IMC $26.39 \mathrm{~kg} / \mathrm{m}^{2}+/-4.65$, la media de menarquia fue de 12.79 años $+/-1$, la media de Periodo Internatal fue de 5.57 años $+/-4.06$, el promedio de estancia en $\mathrm{UCl}$ fue de 4.53

Menos de $20 \%$ de las pacientes admitidas al presente estudio correspondió a menores de 21 años, $74.73 \%$ correspondió a pacientes entre 21 a 40 años de edad. Del total de pacientes admitidas al presente estudio; $50.53 \%$ correspondió a preeclampsia severa de aparición temprana. Un $3.95 \%$ de pacientes admitidas al presente estudio correspondió a pacientes fallecidas.

TABLA 1. comparación de variables cualitativas.

\begin{tabular}{|c|c|c|c|c|c|}
\hline \multirow{2}{*}{ VARIABLE } & \multicolumn{4}{|c|}{ PES TEMPRANA PES TARDIA } & \multirow{2}{*}{$\mathbf{P}$} \\
\hline & $\mathbf{N}$ & $\%$ & $\mathbf{N}$ & $\%$ & \\
\hline \multicolumn{6}{|l|}{ Estado civil } \\
\hline Casada & 29 & 11.74 & 31 & 12.25 & \multirow{4}{*}{0.23} \\
\hline Conviviente & 159 & 64.37 & 177 & 69.96 & \\
\hline Soltera & 58 & 23.84 & 44 & 17.39 & \\
\hline Viuda & 0 & 0 & 1 & 0.40 & \\
\hline \multicolumn{6}{|c|}{ Nivel de instrucción } \\
\hline Iletrada & 41 & 0.41 & 4 & 1.61 & \multirow{5}{*}{0.12} \\
\hline Primaria & 28 & 11.17 & 32 & 12.85 & \\
\hline Secundaria & 164 & 67.77 & 176 & 70.68 & \\
\hline Superior & 34 & 11.41 & 22 & 8.83 & \\
\hline Técnico & 15 & 6.20 & 15 & 6.02 & \\
\hline \multicolumn{6}{|l|}{ IMC* } \\
\hline Infrapeso & 1 & 0.48 & 1 & 0.44 & \multirow{6}{*}{0.93} \\
\hline Normal & 97 & 47.37 & 99 & 42.36 & \\
\hline Sobrepeso & 87 & 34.45 & 72 & 37.99 & \\
\hline Obesidad I & 28 & 11.48 & 24 & 12.23 & \\
\hline Obesidad II & 12 & 4.31 & 9 & 5.24 & \\
\hline Obesidad III & 4 & 1.91 & 4 & 1.75 & \\
\hline Primipaternidad & 95 & 41.48 & 116 & 49.57 & 0.081 \\
\hline \multicolumn{6}{|c|}{ Condición de ingreso } \\
\hline Gestante & 73 & 32.44 & 5 & 1.99 & \multirow[t]{2}{*}{0.000} \\
\hline Puerpera & 152 & 67.56 & 246 & 98.01 & \\
\hline \multicolumn{6}{|l|}{ Tipo de parto } \\
\hline Eutócico & 10 & 5.78 & 32 & 12.55 & \multirow[t]{2}{*}{0.021} \\
\hline Distócico & 163 & 94.22 & 223 & 87.45 & \\
\hline \multicolumn{6}{|c|}{ Sexo del producto } \\
\hline Femenino & 66 & 42.86 & 118 & 50.53 & \multirow[t]{2}{*}{0.144} \\
\hline Masculino & 88 & 57.14 & 116 & 49.57 & \\
\hline \multicolumn{6}{|l|}{ Grupo edad } \\
\hline$<21$ años & 50 & 19.46 & 57 & 22.71 & \multirow{4}{*}{0.49} \\
\hline $21-30$ años & 117 & 45.53 & 98 & 39.04 & \\
\hline 31-40 años & 74 & 28.79 & 81 & 32.27 & \\
\hline$>40$ años & 16 & 6.23 & 15 & 5.98 & \\
\hline
\end{tabular}

IMC* INFRAPESO (<18.5), NORMAL (18.5-24.99), OBESIDAD I (3034), OBESIDAD III (>40) Intervalo de confianza al $95 \%$ 
TABLA 2. Distribución de variables cuantitativas.

\begin{tabular}{|c|c|c|c|c|c|}
\hline \multirow[t]{2}{*}{ Variable } & \multicolumn{2}{|c|}{$\begin{array}{c}\text { PES temprana } \\
(n=332)\end{array}$} & \multicolumn{2}{|c|}{$\begin{array}{l}\text { PES tardía } \\
(\mathrm{n}=325)\end{array}$} & \multirow[t]{2}{*}{$\mathbf{P}$} \\
\hline & MEDIA & IC & MEDIA & IC & \\
\hline \multicolumn{6}{|l|}{ Datos clínicos de la madre } \\
\hline Edad & 27.84 & $26.88-28.81$ & 28.10 & $27.15-29.04$ & 0.72 \\
\hline Peso & 62.85 & $61.14-64.55$ & 62.05 & $60.62-63.48$ & 0.96 \\
\hline Talla & 1.54 & $1.53-1.55$ & 1.54 & $1.53-1.55$ & 0.31 \\
\hline IMC & 26.45 & $25.81-27.09$ & 26.47 & $25.86-27.07$ & 0.87 \\
\hline Menarquia & 12.75 & $12.59-12.91$ & 12.84 & $12.69-12.99$ & 0.39 \\
\hline Edad gestacional & 26.03 & $25.16-26.91$ & 37.20 & $36.94-37.45$ & 0.000 \\
\hline Estancia hospitalaria & 5.01 & $3.92-6.09$ & 3.75 & $3.22-4.28$ & 0.006 \\
\hline PAS & 136.52 & $132.79-140.26$ & 142.98 & $139.25-146.72$ & 0.002 \\
\hline PAD & 85.73 & $83.11-88.34$ & 88.68 & $85.77-91.58$ & 0.07 \\
\hline PAM & 100.16 & 96.63-103.69 & 106.17 & $102.85-109.49$ & 0.005 \\
\hline $\mathrm{FC}$ & 105.50 & $102.28-108.72$ & 102.79 & 99.94-105.65 & 0.25 \\
\hline Índice SOFA & 3.41 & $2.77-4.05$ & 2.58 & $2.04-3.14$ & 0.14 \\
\hline Índice de Shock & 1.13 & $1.06-1.19$ & 1.02 & 0.98-1.07 & 0.03 \\
\hline TVM & 4.16 & $2.67-5.65$ & 2.47 & $1.59-3.36$ & 0.01 \\
\hline \multicolumn{6}{|l|}{ Uso de hemoderivados } \\
\hline Paquete globular & 4.78 & $3.89-5.67$ & 4.31 & $3.75-4.86$ & 0.66 \\
\hline Plaquetas & 7.41 & $6.15-8.66$ & 6.81 & $5.32-8.29$ & 0.26 \\
\hline Plasma fresco congelado & 6.78 & $4.95 \quad 8.61$ & 5.61 & $4.46-6.76$ & 0.35 \\
\hline Crioprecipitado & 8.08 & $6.30-9.86$ & 7.19 & $5.71-8.66$ & 0.30 \\
\hline NHD & 6.36 & $3.14-9.59$ & 5.91 & $3.05-8.77$ & 0.81 \\
\hline \multicolumn{6}{|l|}{ Laboratoriales } \\
\hline Hemoglobina & 9.08 & $8.12-10.04$ & 8.52 & $7.52-9.52$ & 0.000 \\
\hline Plaquetas & 143381 & $132140-154622$ & 137229 & $124135-150323$ & 0.15 \\
\hline Glucosa & 82.38 & $79.32-85.44$ & 81.43 & 79.01-83.85 & 0.81 \\
\hline Albumina & 2.07 & $1.88-2.26$ & 2.08 & $1.81-2.35$ & 0.65 \\
\hline Fibrinógeno & 366.84 & $347.01-386.67$ & 361.94 & $342.18-381.71$ & 0.82 \\
\hline TGO & 237 & $105.02-210.03$ & 239 & $155.30-324.08$ & 0.79 \\
\hline TGP & 132.82 & $85.48-180.14$ & 197.92 & $129.70-27$ & 0.12 \\
\hline BT & 2.10 & $1.57-2.64$ & 2.36 & $1.79-2.93$ & 0.27 \\
\hline $\mathrm{BD}$ & 1.18 & $0.82-1.55$ & 1.28 & $0.89-1.67$ & 0.93 \\
\hline $\mathrm{BI}$ & 1.0 & $0.831-1.17$ & 1.03 & $0.87-1.21$ & 0.45 \\
\hline Protrombina & 5.02 & $4.52-5.51$ & 6.20 & $5.31-7.09$ & 0.20 \\
\hline UREA & 46.22 & $35.02-57.43$ & 37.37 & $31.78-42.96$ & 0.61 \\
\hline Creatinina & 1.68 & $1.36-2.02$ & 1.97 & $1.55-2.41$ & 0.73 \\
\hline Lactato & 2.84 & $1.50-4.19$ & 3.06 & $2.24-3.89$ & 0.02 \\
\hline $\mathrm{FIO} 2$ & 0.48 & $0.43-0.52$ & 0.45 & $0.40-0.49$ & 0.55 \\
\hline $\mathrm{PH}$ & 7.28 & $7.27-7.29$ & 7.29 & $7.27-7.31$ & 0.51 \\
\hline PO2 & 69.42 & $65.92-72.93$ & 68.92 & $64.71-73.13$ & 0.74 \\
\hline $\mathrm{HCO} 3$ & 16.32 & $15.78-16.85$ & 16.84 & $16.34-17.36$ & 0.15 \\
\hline
\end{tabular}

Nivel de significancia $95 \%$

Abreviatura: IMC: índice de masa corporal, PAS: presión arterial sistémica, PAD: presión arterial diastólica, PAM: presión arterial media, FC: frecuencia caardiaca, SOFA: Sequential ORgan Failure Assesment, TGO: transaminasa glutámico-oxalacética, TGP: alanina-aminotransferasa, BT:Bilirrubina Total, BD: Bilirrubina Directa, BI: Bilirrubina Indirecta, TVM: Tiempo Ventilacion Mecanica, NHD: Numero de hemoderivados.

TABLA 3. Distribución de datos del recién nacido de madre preeclamptica

\begin{tabular}{|c|c|c|c|c|c|}
\hline \multirow{2}{*}{ Variable } & \multicolumn{2}{|c|}{$\begin{array}{l}\text { PES temprana } \\
(n=332)\end{array}$} & \multicolumn{2}{|r|}{$\begin{array}{c}\text { PES tardía } \\
(n=325)\end{array}$} & \multirow[t]{2}{*}{$\mathbf{P}$} \\
\hline & MEDIA & IC & MEDIA & IC & \\
\hline Peso rn & 1595.34 & $1493.1-1697.5$ & 2968.77 & $2883.81-3053.74$ & 0.000 \\
\hline Talla rn & 39.88 & $39.13-40.62$ & 47.38 & $46.89-47.86$ & 0.000 \\
\hline APGAR 1er min & 5.07 & $4.61-5.53$ & 7.02 & $6.74-7.30$ & 0.000 \\
\hline
\end{tabular}

Nivel de significancia 95\% Abreviatura: rn: recién nacido 
TABLA 4. Distribución de pes de aparición temprana y tardía en relación a enfermedad hipertensiva del embarazo.

\begin{tabular}{|c|c|c|c|c|c|}
\hline \multirow{2}{*}{$\begin{array}{ll} & \text { EHE } \\
\text { PES }\end{array}$} & \multicolumn{2}{|c|}{ PES TEMPRANO } & \multicolumn{2}{|c|}{ PES TARDIO } & \multirow{2}{*}{$\begin{array}{l}\text { TOTAL } \\
175(67.31 \%)\end{array}$} \\
\hline & 94 & $63.09 \%$ & 81 & $72.97 \%$ & \\
\hline HELLP & 26 & $17.45 \%$ & 15 & $13.51 \%$ & $41(15.77 \%)$ \\
\hline ECLAMPSIA & 25 & $16.78 \%$ & 14 & $12.61 \%$ & $39(15 \%)$ \\
\hline HELLP+ECL & 2 & $1.34 \%$ & 1 & $0.9 \%$ & $39(15 \%)$ \\
\hline HGAE & 2 & 1.34 & 0 & $0.00 \%$ & $2(0.77 \%)$ \\
\hline TOTAL & 149 & $100 \%$ & 111 & $100 \%$ & $260(100 \%)$ \\
\hline
\end{tabular}

Fisher's exact $=0.481$ nivel de significancia 95\%

Abreviatura: EHE: enfermedad hipertensiva del embarazo, HELLP + ECL: síndrome de HELP asociado a eclampsia, HGAE:

En el periodo de estudio que correspondió a dos años (agosto del 2014 al 31 de agosto del 2017) se evaluaron 657 registros; 332 registros de pacientes con diagnóstico de Preeclamsia severa de aparición temprana y 325 pacientes con diagnóstico de preeclampsia severa de aparición tardía. En las pacientes con diagnóstico de Preeclampsia severa de aparición temprana la edad promedio correspondió a 27.84 (IC 26.88- 28.81) años de edad ( $p: 0.72$ ), el peso no tuvo diferencias significativas entre las PES de aparición temprana en relación a las PES de aparición tardía; $62.85 \mathrm{~kg}$ (IC: 61.14-64.55), la talla no tuvo diferencias significativas entre las PES de aparición temprana en relación a las PES de aparición tardía, el IMC no tuvo diferencias significativas entre las PES de aparición temprana en relación a las PES de aparición tardía 26.45 (25.81-27.01), la estancia hospitalaria si tuvo diferencias significativas entre las PES de aparición temprana en relación a las PES de aparición tardía 5.01 días (3.926.09)(p:0.006); el promedio de estancia hospitalaria fue mayor en las pacientes con PES de aparición temprana en relación de las pacientes con PES de aparición tardía. El peso del recién nacido si mostro diferencias significativas entre las PES de aparición temprana en relación a las PES de aparición tardía; 1595.34 cm (IC: 1493.15 -1697.54) ( $p: 0.000)$, la talla del Recién nacido mostro diferencias significativas entra las PES de aparición temprana en relación a las PES de aparición tardía $39.88 \mathrm{~cm}$ (IC: $39.13-$ 40.62)(p:0.000), el APGAR al minuto fue menor en el grupo de PES de aparición temprana 5.07 (IC: 4.61-5.53) (p:0.000), el valor de la Hemoglobina sérica fue mayor en las pacientes con PES de aparición temprana $9.08 \mathrm{mg} / \mathrm{dl}$ (IC: 8.12-10.04)(p:0.000), la PAS fue menor en las PES de aparición temprana en relación a las PES de aparición tardía 136.52mmHg (IC: 132.79-140.26)(p:0.002), la PAM fue menor en las PES de aparición temprana en relación a las PES de aparición tardía 100.16mmHg (96.63-103.69) (p:0.005), el nivel de Lactato es menor en las PES de aparición temprana en relación a las PES de aparición tardía 2.84 (IC: 1.50-4.19), el tiempo de Ventilación Mecánica fue mayor en las PES de aparición temprana en relación a las PES de aparición tardía; 2.84 días (Cl:2.67-5.65)(P:0.01), el Índice de shock (IS) fue mayor en las pacientes con PES de aparición temprana en relación al grupo de PES de aparición tardía. 1.13 (IC: 1.06-1.19)(p:0.03)
En la variable Estado civil la convivencia o unión estable fue la más frecuente tanto en el grupo de PES de aparición temprana así como con la PES de aparición tardía. (64.37\% vs $69.96 \%$ ), respecto al nivel de educación Secundaria es la más frecuente tanto en la PES de aparición temprana como tardía $(67.77 \%$ y $70.68 \%$ ), respecto al IMC el grupo más frecuente fue el IMC normal. (47.37\% y $42.36 \%$ ), respecto a la variable primipaternidad se evidencia que no hay diferencias significativas entre las PES de aparición temprana vs PES de aparición tardía; (41.48\% y 49.57\%), la condición de ingreso si mostro diferencias significativas ( $p: 0.000$ ); donde las puérperas fueron más frecuentes en el grupo de PES de aparición tardía mientras que las gestantes fueron más frecuentes en el grupo de PES de aparición temprana, el tipo de parto también mostro diferencias significativas (0.021) donde el parto distócico fue más frecuente en ambos grupos; PES de aparición temprana y tardía, el sexo del producto no mostro diferencias significativas ( $p$ : 0.144), respecto a la evaluación de la edad recodificada los dos grupos se mostraron homogéneos. ( $p: 0.49)$.

\section{DISCUSIÓN}

La Preeclampsia severa es una entidad frecuente en la Unidad de Cuidados Intensivos Materno (50.19\%), la primera causa de mortalidad materna en la UCI materna del INMP corresponde a complicaciones de la Preeclampsia severa, de hecho, las complicaciones hemorrágicas asociadas a Síndrome HELLP, hemorragias post parto, rotura hepática espontanea son frecuentes.

Se plantea dos entidades distintas como son la PES de aparición temprana y PES de aparición tardía indicando que la primera tendría origen placentario mientras que la segunda tendría origen materno. Lacunza muestra diferencias variadas donde afirma que la PES de aparición temprana tiene mayor asociación con la eclampsia, síndrome HELLP. Sin embargo, en el presente estudio no se observan diferencias y al contrario los grupos relacionados a la enfermedad hipertensiva del embarazo (EHE) son muy similares. 
Lacunza también manifiesta diferencias hemodinámicas, en el presente estudio si se demostró diferencisas significativas entre las PAS y la PAM. Sin embargo, no hubo mayores datos hemodinámicos que permitieran confirmar dichas diferencias.

Respecto al producto es lógico encontrar diferencias en los grupos de PES de aparición temprana y tardía por la misma prematuridad es lógico encontrar productos más pequeños, con menos peso y con APGAR más bajo.

Sería interesante hacer la correlación con las características anatomopatológicas y microscópicas de las placentas de ambos grupos que nos permita afirmar un esbozo de diferencia encontrado en otros estudios.

Es necesario contar con marcadores bioquímicos que nos permitan hacer la evaluación de asociaciones encontrados en otros artículos publicados.

En el presente estudio no se encuentran grandes diferencias respecto a la PES de aparición temprana y tardía; sin embargo, es necesario realizar mayores estudios que incluyan variables fetales, placentarios y de biomarcadores que nos permitan tener una visión más amplia del problema.

En conclusión, no se observa diferencias significativas entre PES de aparición temprana y eclampsia. Los recién nacidos, presentan peso, talla y APGAR menores en el grupo de aparición temprana que el grupo de aparición tardía.

Financiamiento: Concurso de Investigación INMP 2019.

Conflicto de interés: Los autores declaran no tener ningún conflicto de intereses.

\section{REFERENCIAS BIBLIOGRÁFICAS}

1. Armaly Z, Jadaon JE, Jabbour A, Abassi ZA. Preeclampsia: Novel Mechanisms and Potential Therapeutic Approaches. Front Physiol [Internet]. 25 de julio de 2018 [citado 17 de agosto de 2018];9. Disponible en: https://www.ncbi.nlm.nih. gov/pmc/articles/PMC6068263/

2. American College of Obstetricians and Gynecologists, American College of Obstetricians and Gynecologists, editores. Hypertension in pregnancy. Washington, DC: American College of Obstetricians and Gynecologists; 2013. 89 p.

3. Duley L. The global impact of pre-eclampsia and eclampsia. Semin Perinatol. junio de 2009;33(3):130-7.

4. Aouache R, Biquard L, Vaiman D, Miralles F. Oxidative Stress in Preeclampsia and Placental Diseases. Int J Mol Sci [Internet]. 17 de mayo de 2018 [citado 17 de agosto de 2018];19(5). Disponible en: https://www.ncbi.nlm.nih.gov/ pmc/articles/PMC5983711/

5. Paredes ROL, Pacheco-Romero J. Preeclampsia de inicio temprano y tardío: una antigua enfermedad, nuevas ideas. $: 11$.

6. Raymond D, Peterson E. A critical review of early-onset and late-onset preeclampsia. Obstet Gynecol Surv. agosto de 2011;66(8):497-506.

7. Lisonkova S, Joseph KS. Incidence of preeclampsia: risk factors and outcomes associated with early- versus lateonset disease. Am J Obstet Gynecol. 1 de diciembre de 2013;209(6):544.e1-544.e12.

8. Sulistyowati S. Early and Late Onset Preeclamsia: What did really Matter? J Gynecol Womens Health [Internet]. 27 de junio de 2017 [citado 19 de agosto de 2018];5(4). Disponible en: https://juniperpublishers.com/jgwh/JGWH.MS.ID.555670.php

\section{Correspondencia:}

Walter De la Peña Meniz

Dirección: Jr. Santa Rosa 941 Lima

Correo:wdlpmeniz@gmail.com

Teléfono: 996014918 